\title{
Occult axial involvement in patients with psoriatic arthritis mutilans: a case report
}

\author{
F.M. Perrotta, S. Scriffignano, A. De Socio, E. Lubrano
}

Academic Rheumatology Unit, Dipartimento di Medicina e Scienze della Salute Vincenzo Tiberio, Università degli Studi del Molise, Campobasso, Italy

\section{SUMMARY}

We present the case of a patient with psoriatic arthritis (PsA) mutilans and occult axial involvement. The patient had suffered from PsA mutilans for more than a decade, with severe residual articular damage, but had been in clinical remission for years. Clinical axial involvement was never reported; however, magnetic resonance imaging of the sacroiliac joints, performed for other reasons, documented active inflammation and damage even without clinical symptoms. These findings corroborated the hypothesis that axial involvement could be asymptomatic, subclinical and, furthermore, underdiagnosed or even occult in patients with PsA mutilans, in which cases it should be carefully evaluated.

Key words: Psoriatic arthritis; arthritis mutilans; axial involvement; magnetic resonance imaging.

Reumatismo, 2019; 71 (2): 88-91

\section{INTRODUCTION}

P soriatic arthritis (PsA) is a complex and multifaceted inflammatory disease characterized by the development of arthritis (with peripheral or axial joint involvement), enthesitis and dactylitis, in subjects with a personal or familiar history of psoriasis (1).

Moll and Wright first defined PsA as psoriasis associated with inflammatory arthritis (peripheral arthritis and/or spondylitis) usually with a negative serologic test for rheumatoid factor. Because of the variability of clinical presentations of PsA, they suggested a classification of PsA that considered 5 predominant patterns: asymmetric oligoarthritis, symmetric polyarthritis similar to rheumatoid arthritis, spondylitis, distal interphalangeal joint arthritis, and arthritis mutilans. PsA mutilans (PAM) is considered the most severe form of PsA, affecting only a small rate $(5 \%)$ of PsA patients. Although the occurrence of arthritis mutilans associated with PsA is often described as a relatively rare event, the impact on quality of life is great, because of the severity of articular damage $(2,3)$.

Axial involvement in PsA (axPsA) is more common; however, the definition and measurement of axial disease remains problematic. AxPsA can be frequent, ranging between $25 \%$ (early disease and clinical assessment only) and 75\% (late disease and sophisticated imaging) of patients (4). Most of the time, an asymmetrical peripheral arthritis, with or without enthesitis, associated with axial involvement is the commonest presentation of the disease, but some reports showed an association with mutilans subset (3).

\section{CASE REPORT}

We present a case report of a 61-year-old male subject with PAM involving the $3^{\text {rd }}$ finger of the right foot.

\section{Patient history}

The patient was admitted to our Unit on June 2011 with a history of mild scalp psoriasis. The patient had suffered since January 2011 from an acute onset of arthritis in both knees and the metatarsal joints of both feet. Furthermore, the patient reported no history of other relevant clinical complaints. After only six months, the patient developed a telescopic deformation of the 

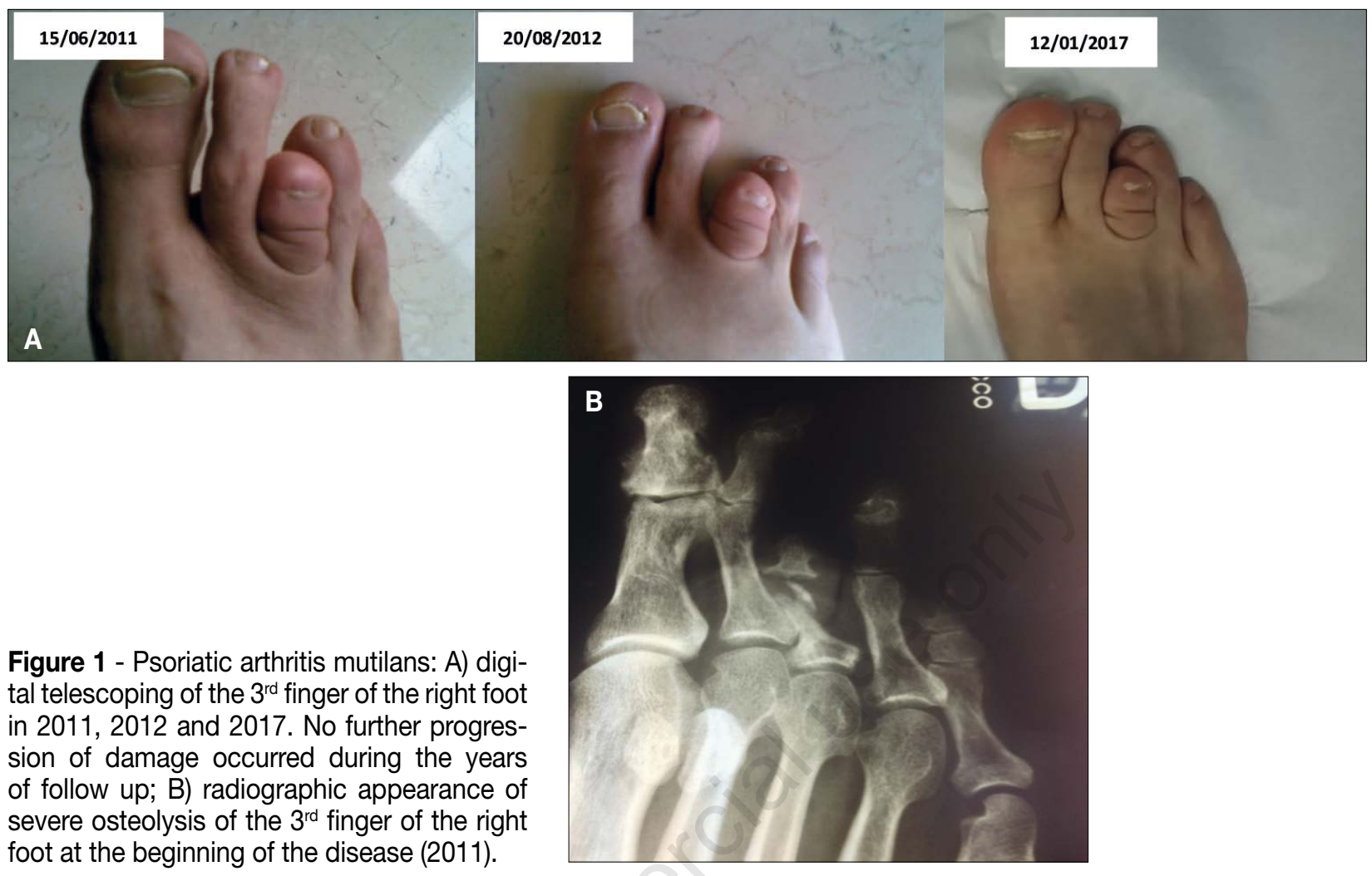

Figure 1 - Psoriatic arthritis mutilans: A) digital telescoping of the $3^{\text {rd }}$ finger of the right foot in 2011, 2012 and 2017. No further progression of damage occurred during the years of follow up; B) radiographic appearance of severe osteolysis of the $3^{\text {rd }}$ finger of the right foot at the beginning of the disease (2011).

$3^{\text {rd }}$ finger of the right foot. At clinical examination, tenderness and swelling of both knees was found. Furthermore, tenderness and swelling of the $2^{\text {nd }}$ and $3^{\text {rd }}$ metatarsal and interphalangeal joints of the right foot was seen, with a telescopic deformity of the third finger (Figure 1A). Pain was very severe - enough to prevent the patient from walking. No signs of enthesitis or dactylitis was objectivated. The patient did not report any history of back pain. Serum samples revealed the presence of raised $C$ reactive protein - CRP (12 mg/L) while erythrocyte sedimentation rate $(\mathrm{ESR})$ was $30 \mathrm{~mm} / \mathrm{h}$. Rheumatoid factor (nephelometry) was negative and anti-citrullinated proteins antibodies were absent. Radiographic assessment of the right foot demonstrated the presence of a severe osteolysis with neardisappearance of the middle phalanx of third finger (Figure 1B). Skin involvement was very limited (only a small lesion of the scalp and the $2^{\text {nd }}$ and $3^{\text {rd }}$ fingers of the right hand with nail pitting) with a psoriasis area severity index (PASI) of 0.4 .
The patient was treated with NSAIDs and subcutaneous methotrexate (MTX) $15 \mathrm{mg}$ weekly. After only six months of MTX therapy, resolution of both knees and metatarsal arthritis with normalization of laboratory parameters and induction of a remission state was observed. After 3 years, the patient suspended all treatments because of sustained remission and he was followed up yearly until 2017. At all examinations, the tender and swollen joints count were 0 , VAS pain and disease activity was $0 \mathrm{~cm}$ $(0-10)$ and no enthesitis or dactylitis were reported by the patient. The HAQ score ranged from 0 to 0.5 at each visit despite the presence of residual damage in the $3^{\text {rd }}$ finger. On January 2018, the patient was admitted to our outpatient clinic for a routine follow-up. Sustained remission (minimal disease activity according to Coates et al. 7/7 criteria) (5) was reported. Laboratory parameters were also normal. However, the patient underwent an $\mathrm{x}$-ray of the pelvis and complete spine for other reasons two months before the visit (the patient was re- 



Figure 2 - MRI (T1 and STIR sequences of sacroiliac joints). Bone marrow oedema is present on both iliac and sacral sites of the left sacroiliac joint in the STIR sequences (white arrows). Erosions and pseudo-enlargement of left sacroiliac joints (red arrows) are visible in T1 sequences.

ferred for a potential mechanical pain only in the cervical spine, developed after some days of intense work activity and which disappeared after a few days of NSAIDs treatment). A suspected sacroiliitis (grade 1) was found, in the absence of clinical symptoms of back pain. Moreover, the patient reported no buttock pain or any other symptoms potentially linked to a possible axial involvement. At clinical examination, the Shober test was normal $(6 \mathrm{~cm})$ and the Bath Ankylosing Spondylitis Metrology Index was 1 . Therefore, according to this suspected $\mathrm{x}$-ray examination, an MRI scan of sacroiliac joints was performed, in agreement with the patient. MRI assessment showed the presence of bone marrow edema, suggesting an active sacroiliitis and bone damage at the level of the left joint, as shown in both STIR and T1 sequences (Figure 2). Despite the presence of active axial inflammation, no treatment was introduced because of the persistence of clinical remission.

\section{DISCUSSION}

Estimates of prevalence of PAM in PsA cohorts range from $0.6 \%$ to $21 \%$ but it seems to be usually closer to $5 \%$, as originally described by Moll and Wright. A populationbased study in adult Nordic populations estimates a prevalence of 3.69 per million, with similar sex distribution (6), but the studies performed on this intriguing clinical subset are scarce. Furthermore, the definition of PAM could be problematic because the lack of accepted classification criteria, with different definitions of PAM found in literature (7). In the Nordic PAM study, the most frequent joints that showed PAM were the distal interphalangeal joint (DIP) joints on the fourth toe on the right foot and the DIP joint of the second toe of the left foot followed by the left thumb IP joint, the DIP joint of the fifth finger on the left hand, and both DIP joints of the fifth toes and, interestingly, most of the patients had a limited skin involvement. Our patient was in line with the classical PAM patient, with involvement of the $3^{\text {rd }}$ finger of the right foot. Furthermore, a recent review showed that psoriatic nail dystrophy was more prevalent in PAM cases than non-PAM cases and PAM cases were more likely to have radiographic axial disease and radiographic sacroiliitis than non-PAM cases (8).

To our knowledge, no targeted studies were performed to specifically evaluate the outcome and response to treatment of PAM patients. In 2009 a case series of 3 PAM patients treated with anti-TNF (etanercept) was published. The authors showed the effectiveness of these drugs in the induction of a sustained clinical response, although irreversible deformity cannot be treated (9). Our patient had a very good outcome with the use of MTX treatment, which induced a long-term drug-free clinical remission of symptoms and signs and normalized the 
laboratory indices, suggesting that early treatment could prevent other damages. Despite the presence of clinical remission, in our patient, a subclinical axial or even an occult involvement persisted. This is in keeping with previous descriptions of occult types of PsA (10) and suggesting that axPsA is an intriguing aspect of the disease (11). Moreover, the patient had been in clinical remission for peripheral involvement for years and the occurrence of an axial involvement reinforced the need for appropriate imaging studies in PsA patients (12). However, the development of an axial involvement may suggest modifying the treatment scheme, according to the literature (13). We demonstrated that is possible to achieve MDA in patients with axPsA treated with anti-TNF $(14,15)$.

Finally, this aspect raised some questions as to whether treatment should be continued even in patients who are in remission, or, on the other hand, if the presence of MRI showing inflammatory lesions may be predictive for a reactivation of disease activity and should be treated in all cases. In fact, the concept of treatment to target or targeting to treatment is still a question open to debate in PsA.

\section{CONCLUSIONS}

PAM is a rare subset of PsA that can cause severe deformity and reduction in quality of life. Axial involvement is present in patients with PAM and our case showed that even in a patient with long-term clinical remission, signs of active disease could be present. This case might improve awareness of this intriguing disease and stimulate further studies on a large cohort of patients.

\section{REFERENCES}

1. Ritchlin CT, Colbert RA, Gladman DD. Psoriatic arthritis. N Engl J Med. 2017; 376: 957-70.
2. Moll JM, Wright V. Psoriatic arthritis. Semin Arthritis Rheum. 1973; 3: 55-78.

3. Haddad A, Chandran V. Arthritis mutilans. Curr Rheumatol Rep. 2013; 15: 321.

4. Lubrano E, Parsons WJ, Marchesoni A, et al. The definition and measurement of axial psoriatic arthritis. J Rheumatol Suppl. 2015; 93: 40-2.

5. Coates LC, Helliwell PS. Defining low disease activity states in psoriatic arthritis using novel composite disease instruments. J Rheumatol 2016; 43: 371-5.

6. Gudbjornsson B, Ejstrup L, Gran JT, et al. Psoriatic arthritis mutilans (PAM) in the Nordic countries: demographics and disease status. The Nordic PAM study. Scand J Rheumatol. 2013 ; 42: 373-8.

7. Haddad A, Johnson SR, Somaily M, et al. Psoriatic arthritis mutilans: clinical and radiographic criteria. A Systematic Review. J Rheumatol. 2015; 42: 1432-8.

8. Jadon DR, Shaddick G, Tillett W, et al. Psoriatic arthritis mutilans: characteristics and natural radiographic history. J Rheumatol. 2015; 42: 1169-76.

9. Pomerantz RG, Mody E, Husni ME, Qureshi AA. Follow-up of psoriatic arthritis mutilans patients treated with anti-TNF-alpha therapy. J Drugs Dermatol. 2009; 8: 406-12.

10. Palazzi C, Lubrano E, D'Angelo S, Olivieri I. Beyond early diagnosis: occult psoriatic arthritis. J Rheumatol. 2010; 37: 1556-8.

11. Lubrano E, Spadaro A. Axial psoriatic arthritis: an intriguing clinical entity or a subset of an intriguing disease? Clin Rheumatol. 2012; 31: 1027-32.

12. Spadaro A, Lubrano E. Psoriatic arthritis: imaging techniques. Reumatismo. 2012; 64: 99106.

13. Nash P, Lubrano E, Cauli A, et al. Updated guidelines for the management of axial disease in psoriatic arthritis. J Rheumatol. 2014; 41: 2286-9.

14. Lubrano E, Parsons WJ, Perrotta FM. Assessment of response to treatment, remission, and minimal disease activity in axial psoriatic arthritis treated with tumor necrosis factor inhibitors. J Rheumatol. 2016; 43: 918-23.

15. Lubrano E, Perrotta FM, Marchesoni A, et al. Remission in nonradiographic axial spondyloarthritis treated with anti-tumor necrosis factor- $\alpha$ drugs: an Italian multicenter study. J Rheumatol. 2015; 42: 258-63. 